\title{
A comparison of tactile spatial sensitivity on the palm and fingerpad
}

\author{
JAMES C. CRAIG and KEITH B. LYLE \\ Indiana University, Bloomington, Indiana
}

\begin{abstract}
Studies of tactile spatial pattern perception have, for the most part, been carried out using the fingerpad. On the basis of these studies, models have been developed linking spatial pattern identification and resolution with underlying neural structures. It has been suggested that with appropriate scaling, these models would apply to the processing of spatial patterns presented to other sites on the body. Spatial sensitivity was examined on another site on the body, the palm, using two measures, letteridentification and grating orientation. The results from these measures were compared with results from similar studies conducted on the fingerpad and with estimates of the density of innervation of the fingerpad and palm. To produce levels of performance similar to those on the fingerpad required letters on the palm $50 \mathrm{~mm}$ in height, seven to nine times larger than those used on the fingerpad. Gratings had to be six to more than seven times larger on the palm to produce the same levels of performance achieved on the fingerpad. For the two types of receptor systems sensitive to spatial information, the ratio of density of innervation between the fingerpad and the palm is estimated to be $5.7: 1$ and 8.8: 1 . Performance of spatial tasks on the palm can be predicted quantitatively from fingerpad data with a moderate degree of accuracy. Qualitative comparisons between the palm and fingerpad data indicate that spatial patterns are processed similarly at the two sites.
\end{abstract}

Beginning with Weber's (1834/1996) work on the twopoint threshold, a major aim of studies of tactile spatial sensitivity has been to correlate psychophysical results with underlying neural structures. Initially, these attempts were qualitative in nature, but recent neurophysiological and psychophysical studies have allowed more quantitative comparisons to be made. Neurophysiological studies have clarified the nature and functioning of mechanoreceptors, particularly in glabrous skin. Several lines of evidence point to the existence of four types of peripheral fibers that respond to mechanical stimuli (Bolanowski, Gescheider, Verrillo, \& Checkosky, 1988). The fiber types are classified according to their responses to transient and sustained stimuli and to the size of their receptive fields. Two of the four types of fibers convey spatial information, slowly adapting Type I (SAI) and rapidly adapting (RA). The fiber types, presumed mechanoreceptors (Merkel disks for SAI fibers and Meissner corpuscles for RA fibers), and central connections are referred to as the SAI and RA receptor systems. Although both neurophysiological and psychophysical evidence supports the view that the SAI and RA systems are responsible for conveying spatial information, it appears that the finest spatial patterns are conveyed by the SAI

This research was supported by the National Institutes of Health Grant DC00095, National Institute on Deafness and Other Communication Disorders. We thank Roger Rhodes for his assistance in these experiments. Correspondence should be addressed to J. C. Craig, Department of Psychology, 1101 E. 10th St., Indiana University, Bloomington, IN 47405-7007 (e-mail: craigj@indiana.edu). system (Greenspan \& Bolanowski, 1996; Johnson \& Hsiao, 1992; Johnson, Hsiao, \& Twombly, 1995; Phillips \& Johnson, 1981).

These studies of the functioning of the SAI and RA systems can be combined with estimates of the density of innervation to provide quantitative predictions about spatial resolution. Investigators-Johansson and Vallbo (1979) with human subjects and Darian-Smith and Kenins (1980) with monkeys-have classified, counted, and estimated the number and type of nerve fibers innervating the glabrous skin of the finger and, with human subjects, portions of the hand as well. On the basis of these results, investigators have estimated that the density of innervation is approximately one afferent per square millimeter for both SAI and RA systems (Johnson et al., 1995).

One of the aims of the present study was to compare neurophysiological results with measures of spatial sensitivity. The two-point threshold has been widely used in past studies; however, several recent articles have discussed the problems with the two-point threshold as a measure of spatial resolution (Craig \& Johnson, 2000; Johnson, Van Boven, \& Hsiao, 1994). These articles have pointed out that there are two forms of the two-point threshold, subjective and objective. The subjective form is the more commonly used form. In it, subjects are presented two points of stimulation and asked to judge whether they feel two points or a single point. This method is subject to serious criterion problems and extreme within- and between-subjects unreliability. In one study the threshold for responding "two" decreased by more than a factor of five over the course of a month of testing. 
The situation is not improved by the objective form, in which subjects judge whether one or two points were presented to the skin. Here the threshold can be shown to be zero, implying infinite spatial resolution (Craig \& Johnson, 2000).

The measure of spatial resolution that we used was grating orientation. In this measure, subjects are presented with a grating in one of two orientations on the skin. For example, on the fingerpad the gratings are presented in either a proximal-distal orientation or at right angles to that in the lateral-medial orientation. The subject indicates the orientation. The width of the grooves is varied to determine a psychometric function. To achieve $75 \%$ correct levels of performance requires a groove width of approximately $1 \mathrm{~mm}$ on the fingerpad (Johnson \& Phillips, 1981; Sathian, Zangaladze, Green, Vitek, \& DeLong, 1997). Grating orientation has also been used to measure sensitivity on the face (Patel, Essick, \& Kelly, 1997; Sathian \& Zangaladze, 1996; Van Boven \& Johnson, 1994a, 1994b) and on several regions of the hand (Craig, 1999). In general the results using this measure have shown reasonable correspondence with what is known about the density of innervation for the locations tested.

Quite a different task, letter identification, has also been used to measure spatial sensitivity on the fingerpad. The grating orientation task relies on devising stimuli that are identical except for the orientation in which they are presented to the skin. The letter identification task uses a large number of stimuli that vary in both intensive and spatial dimensions. It has been reasoned that the number of patterns that must be identified precludes subjects using intensive cues and that subjects must rely on spatial cues (Johnson \& Phillips, 1981). If the set of patterns were limited to two or three letters, subjects might well use intensive cues as a basis for their decision. Subjects can identify about three levels of intensity with a high degree of accuracy (Geldard, 1960). If subjects can identify individual letters from a set of 26 with a reasonable degree of accuracy, it is likely that they are doing it on the basis of spatial information.

Johnson and Phillips (1981) measured both letter identification and grating orientation sensitivity on the fingerpad and compared quantitatively the two measures to one another. Further, they predicted the psychophysical results based on the estimates of the density of innervation. They reasoned that if both letter identification and grating orientation tap spatial mechanisms, then sensitivity as determined by the two methods ought to be similar. Johnson and Phillips assumed that letters could reasonably be represented by a $5 \times 5$ neural matrix, that is, when the height of the letter was approximately equal to the overall distance between five neural units. They also reasoned that gratings could be resolved when the period of the grating (the distance equal to the groove width plus the ridge width or, in other words, twice the groove width) was equal to the spacing of two neural units.

Johnson and Phillips (1981) generated psychometric functions for both grating orientation (percent correct as a function of spatial period in millimeters) and for letter identification (percent correct as a function of letter height in millimeters). They found agreement between their letter identification data and their grating orientation results when the two were scaled in the ratio of 5:2. In subsequent studies and in the present study, grating orientation results have been expressed in terms of the width of the groove rather than the period. Because the ridge width and groove width are identical, the groove width is equal to half the period of the grating. Thus, the letter height needed for reasonable levels of performance should be about five times the groove width that can be resolved. What constitutes a "reasonable" level of performance? Johnson and Phillips used performance levels halfway between chance and perfect performance: For grating orientation this is $75 \%$ correct, for letter identification $52 \%$ correct (chance equals $3.8 \%$ ). When scaled appropriately, grating orientation performance and letter identification are similar when compared at these two levels of performances. On the basis of these and other results, Johnson and his coworkers (Johnson \& Hsiao, 1992; Johnson et al., 1995) have developed a model of spatial pattern perception based on the quality of the peripheral neural representation.

Loomis (1990) has elaborated on this model with specific reference to visual pattern identification. Both Johnson and Loomis agree that the initial stage in processing tactile spatial patterns involves a low-pass filter. To compare visual and tactile processing, one needs to either equate for the size of the patterns (very small visual patterns and large tactile patterns, Phillips, Johnson, \& Browne, 1983) or low-pass filter the visual patterns (Loomis, 1990). With these manipulations, performance should be equivalent in the two modalities. In a series of studies, Loomis $(1981,1982,1990)$ measured tactile pattern identification and compared it with visual pattern identification of the same set of patterns. The visual patterns were low-pass filtered by blurring them. Phillips et al. achieved similar levels of tactile and visual performance by using very small visual letters. Both procedures produced similar results in that the correlation was high between letters correctly identified either visually or tactually. Also, the types of confusions were similar for both the visual and tactile patterns.

Loomis (1990) goes on to characterize a second stage of processing. This stage involves a comparison of the transformed stimulus (low-pass filtered) with templates of the patterns in the set. Of particular importance for the present study is the fact that Loomis makes an explicit prediction about character recognition at locations on the body other than the fingerpad: "It is fair to suppose that character recognition is functionally equivalent at different body loci when the stimuli are scaled in size at each site in relation to the spatial resolution at that site" (p. 118).

Nearly all of the results in support of these models of spatial pattern identification have come from studies using the distal fingerpad. The fingerpad has been used in most of the studies of spatial acuity because it is one of the most sensitive areas on the body and because it is the site 
that is most heavily involved in haptic exploration. As noted, Loomis (1990) has suggested that tactile character recognition ought to be the same at other sites as that observed on the fingerpad, provided that the stimuli are adjusted in size appropriately. Also, grating orientation appears to be an appropriate measure of spatial acuity and ought to vary in the same way as letter identification performance.

In the present study we used previous measures of spatial acuity obtained on the distal fingerpad and compared these measures with those obtained on another site. Our aim was to measure spatial acuity using letter identification and grating orientation tasks at a site for which there were estimates of the density of innervation. The number of such possible sites is limited. Density of innervation estimates exist for only three sites - the fingertip, the rest of the finger, and the palm. From grating orientation measurements made in a recent study (Craig, 1999), it appears that the size of letters needed to achieve reasonable levels of performance on the more proximal fingerpads would be too large for the surface area of the pad. Thus, the palm was selected as the site of stimulation. Johansson and Vallbo (1979) estimated that the ratio of the density of innervation from the fingerpad to the palm for the SAI system is 8.8:1 and for the RA system is 5.7:1. From these estimates, it appears that letters would have to be six to nine times larger when presented to the palm relative to the fingerpad to achieve the same levels of performance. For gratings this would be $6-9 \mathrm{~mm}$ or more in width. From earlier studies, we estimated letters varying in height from 5.5 to $7.2 \mathrm{~mm}$ should produce levels of performance from $54 \%$ to $60 \%$ correct on the fingerpad (Johnson \& Phillips, 1981; Loomis, 1981; Phillips et al., 1983). We used letters $50 \mathrm{~mm}$ in height, 6.9-9 times larger than the fingerpad letters.

The present study consisted of two sets of measurements, grating orientation sensitivity (Experiment 1) and letter identification (Experiment 2). Both types of measurements were carried out on the palm. In addition, grating orientation sensitivity was also measured on the fingerpad. The main question was whether spatial pattern information is processed similarly at two sites that differ substantially in sensitivity. Specifically, these measurements addressed the following questions: Can grating orientation performance on the palm relative to the fingerpad be predicted by the changes in density of innervation between the two sites? Can letter identification performance comparable to that obtained on the fingerpad be obtained on the palm when letters are scaled appropriately in size? Does grating orientation performance on the palm predict letter identification performance?

\section{EXPERIMENT 1}

Grating orientation performance was measured on the palm. Measurements were made both before and after subjects received training on a letter identification task (Experiment 2). For purposes of comparison, measurements of grating orientation performance were also made on the fingerpad.

\section{Method}

Subjects. The subjects were 6 Indiana University students, who were paid for their participation. Four females and 2 males participated in the experiment.

Stimuli. For the grating orientation task, domed, circular contactors with square-wave gratings were used. For the palm measurements, the contactors were machined at Indiana University for use in the experiment. Each contactor has a series of alternating, equal-width grooves and ridges cut into it. The depth of the grooves is such that the skin does not touch the bottom of the groove. The diameter of the contactors was $50 \mathrm{~mm}$. Contactors with grooves of four different widths were used: $10,8,6$, and $4 \mathrm{~mm}$. Two sets of the four contactors were machined; the gratings of the one set were offset from the gratings of the other set by one-half cycle. We wanted to reduce the possibility that subjects might use a cue such as where a particular ridge or groove contacted the palm. By varying the gratings by one-half cycle, the grooves and ridges contacted the skin at different locations. The two sets of contactors were used on alternate blocks of trials and produced identical performance. For the fingerpad measurements, a commercially available set of eight domes was used (JVP Domes, Stoelting). The grooves varied in width from 3.0 to $0.35 \mathrm{~mm}$.

Apparatus. In the grating orientation task, the contactors were presented to the palm by means of a weighted shaft. The contactor was attached to one end of the shaft and weights were attached to the opposite end. The combination of the weights, the shaft, and the contactor produced a force of $190 \mathrm{~g}$. The shaft was held in a metal arm such that the shaft could be smoothly raised and lowered. When threshold was measured on the fingerpad, a force of $100 \mathrm{~g}$ was used.

Procedure. Subjects were tested individually. The subject was seated with his/her right arm extended. The hand was positioned on a padded shelf with the palm facing up. The apparatus was aligned so that the stimuli contacted the skin on the center of the palm. The contactor was lowered onto the palm with a force of $190 \mathrm{~g}$. The contactor remained in contact with the skin until the subject responded, generally in 1 or $2 \mathrm{sec}$. The subject was instructed not to move his/her arm or hand so that there was no lateral movement of the contactor across the palm. Subjects kept their eyes closed during testing.

The contactor was presented with the grating in either the proximal-distal (PD) or the lateral-medial (LM) orientation. Subjects responded by indicating the orientation of the grating - proximal or lateral, respectively. Four blocks of trials were completed in each testing session. One groove width was tested during each block of trials. The following groove widths were presented, in order, in each session: $10,8,6$, and $4 \mathrm{~mm}$. On approximately one-half of the trials the contactor was presented in the PD orientation, and on the other half the LM orientation was presented. The order of presentation was determined randomly. Subjects were tested for four sessions. Following letter identification training (Experiment 2), subjects were again tested for four sessions on the grating orientation task. Five of the six subjects were also tested for four sessions on the fingerpad using the same procedure as that used on the palm.

Before each block of trials, subjects performed four practice trials with the contactor that was to be tested in that block of trials and were given feedback as to the correctness of their responses. During the experimental trials, no feedback was provided.

\section{Results}

The percentage correct is plotted as a function of the $\log$ of the groove width (Figure 1). Both the initial and final measurements are shown. Data for all 6 subjects are presented. Two of the 6 subjects were close to chance for both sets of measurements. Posttraining performance is significantly better than pretraining performance. An analysis of variance (ANOVA) showed a significant effect of pre-/postcondition $[F(1,5)=12.32, p<.05]$, a 


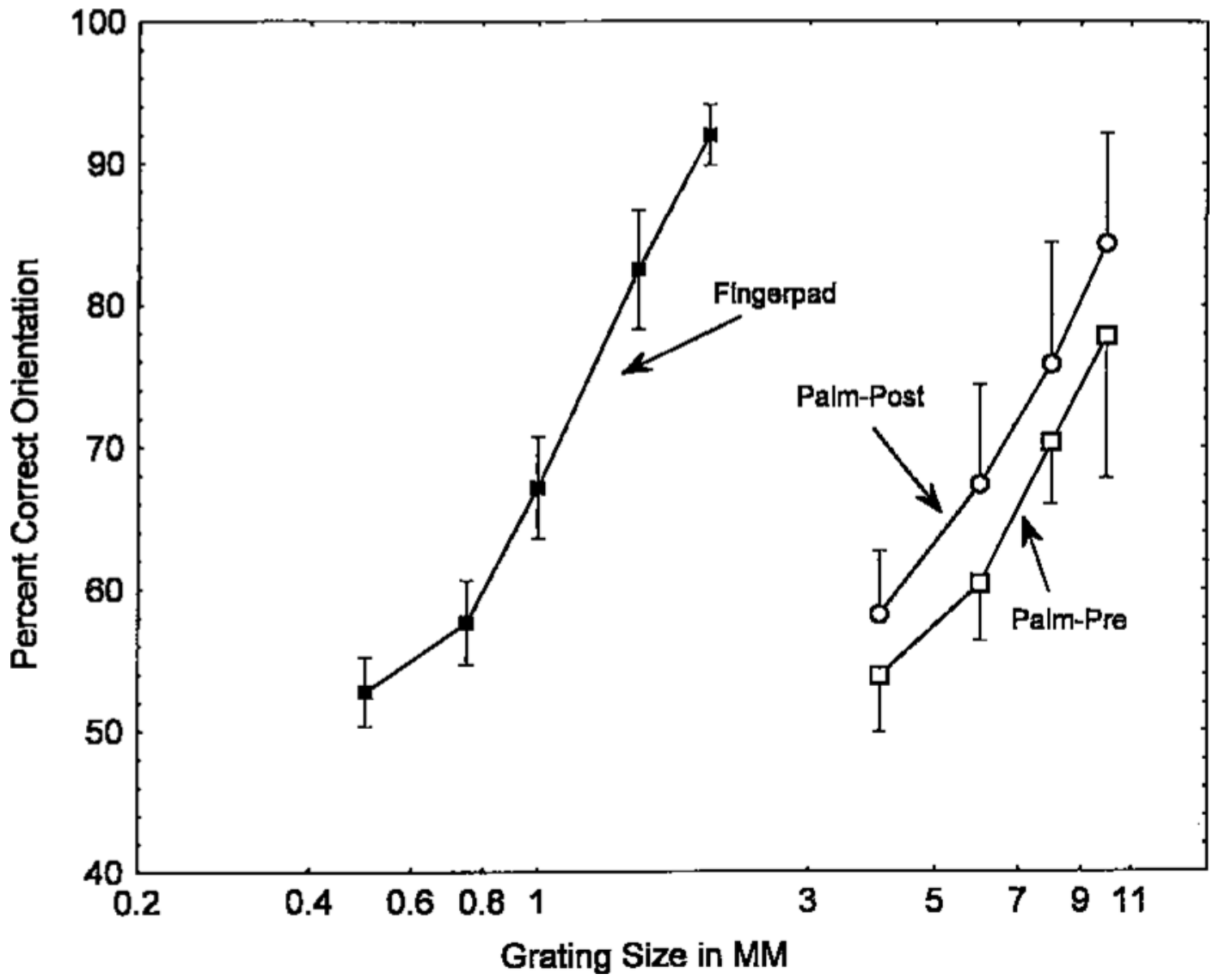

Figure 1. Percent correct orientation as a function of groove width. Results are from testing both the fingerpad and the palm. The palm-pre function represents average performance prior to any training on the letter identification task. The palm-post function represents the average of performance following training.

significant effect of groove width $[F(3,15)=8.11, p<$ $.01]$, but no significant interaction $[F(3,15)=0.07, p>$ .05] The $75 \%$ correct point, which is typically taken as threshold, is $9.2 \mathrm{~mm}$ for the initial measurements and drops to $7.8 \mathrm{~mm}$ for the final measurements. The issue of the possible mechanisms that might account for the change in sensitivity between initial and final measurements is examined in the General Discussion. Also, shown for purposes of comparison is the psychometric function resulting from measurements made on the fingerpad. These data were obtained from 5 of the 6 subjects tested on the palm. The threshold for the fingerpad is $1.25 \mathrm{~mm}$.

\section{Discussion}

Depending upon whether one uses the initial or final threshold estimates from the palm, the ratio of sensitivity between palm and fingerpad is either $7.4: 1$ or $6.2: 1$. As noted earlier, the estimated ratio of the density of innervation between fingerpad and palm for the SAI system was 8.8:1 and for the RA system was 5.7:1. Thus, the ratios of sensitivity are within the range predicted by the density of innervation.
A major issue is whether spatial patterns are processed similarly when presented to different sites on the body. Van Boven and Johnson (1994a) measured grating orientation on the lip, tongue, and fingerpad. The thresholds (75\% correct) were $0.51 \mathrm{~mm}$ on the lip, $0.58 \mathrm{~mm}$ on the tongue, and $0.98 \mathrm{~mm}$ on the fingerpad, a range of sensitivity of about 2:1. The psychometric functions for the three sites were parallel to one another. Van Boven and Johnson (1994a) concluded that the results indicated that spatial information is processed similarly at the three sites and that neural mechanisms for these sites are similar. The change in sensitivity from the fingerpad to the palm is considerably greater, with the palm six to seven times less sensitive. Loomis and Lederman (1986) have suggested that plotting data from different body sites as a function of the log of the spatial dimension should result in parallel functions. The palm functions shown in Figure 1 do appear to parallel the fingerpad function. To demonstrate this similarity more clearly, we replotted the fingerpad data, multiplying each data point by 7.4 to compare with the pretesting function, and plotted this result in Figure 2. The fingerpad data were replotted again 


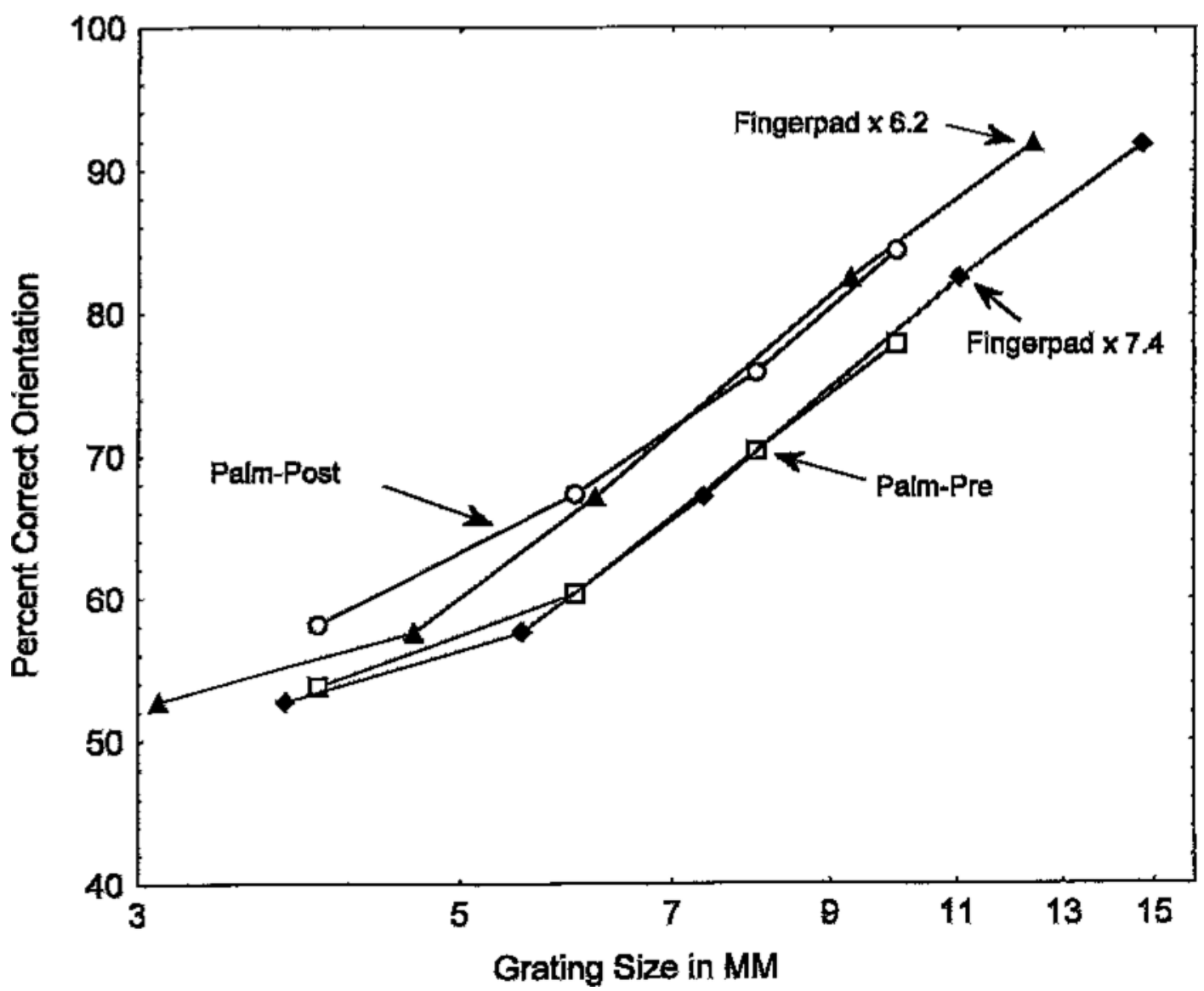

Figure 2. Percent correct orientation as a function of groove width. Each data point on the fingerpad function from Figure 1 is replotted and multiplied by two different constant values, 7.4 and 6.2. See text for details. The palm-pre and palm-post functions from Figure 1 are presented for comparison.

after multiplying each data point by 6.2 to compare with the posttesting function. The similarity of the fingerpad and palm functions indicates that even with fairly major changes in sensitivity the neural mechanisms underlying spatial acuity on glabrous skin remain the same.

As noted, there is strong evidence that SAI fibers are responsible for processing fine spatial information. SAI fibers can convey information about gratings down to widths of approximately $1 \mathrm{~mm}$, whereas RA fibers do not respond to gratings less than $3 \mathrm{~mm}$ in width (Phillips \& Johnson, 1981). Thus, grating orientation sensitivity on the fingerpad likely depends upon activity in the SAI system. It is less clear which receptor systems are responsible for conveying spatial information on the palm. With grating thresholds greater than $4 \mathrm{~mm}$, it is likely that spatial information could be carried by both receptor systems. The estimates of neural density are probably not precise enough and the psychophysical results not unambiguous enough to state which one of the two receptor systems is conveying the spatial information. However, it does appear that the two systems are not combining their outputs to achieve greater spatial sensitivity. If the output of the
SAI and RA systems were being combined to improve sensitivity, the effective density of innervation would nearly double on the palm. The grating orientation thresholds would be considerably lower than were in fact obtained.

\section{EXPERIMENT 2}

In Experiment 2, subjects were trained to identify letters presented to the right palm. The major question was whether grating orientation sensitivity, as measured in Experiment 1 , would predict the performance levels achieved in this quite different measure of spatial sensitivity. Following extensive training with the right palm, letter identification was tested on the left palm to see the extent to which improvements in performance were specific to the site at which training was conducted.

\section{Method}

Subjects. The same subjects who participated in Experiment 1 served as subjects in Experiment 2.

Stimuli. The letters used in this task were $50 \mathrm{~mm}$ in height and varied in width from $7 \mathrm{~mm}$ (the letter "I") to $63 \mathrm{~mm}$ (the letter "W"). 


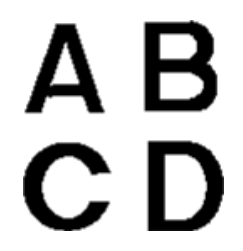

Figure 3. Representations of the letters used in the identification task.

The stroke width for the letters was $7 \mathrm{~mm}$. Each letter was $7 \mathrm{~mm}$ thick. Representations of several of the letters are shown in Figure 3.

Apparatus. A device was constructed to present letters to the palm. The device consisted of a platform on which the subject rested his/her palm. The palm was positioned over a square opening, $7 \times 8 \mathrm{~cm}$. Beneath the opening was a holder in which raised plastic letters were placed. A solenoid was mounted beneath the holder. When the solenoid was activated, it raised the letter vertically through the opening in the platform, and the letter contacted the palm. The letters were placed on a flat, circular plate that was rotated by the experimenter. After each trial the plate was positioned to bring a new letter in place over the solenoid and beneath the subject's palm.

Procedure. Letters were presented one at a time to the subject's right palm. The letter was raised by the solenoid and brought into contact with the subject's palm. At the start of the experiment, each letter was presented for $250 \mathrm{msec}$. After some initial testing, the duration of contact was increased to $1 \mathrm{sec}$. Other investigators have allowed contact times up to several seconds (Loomis, 1990; VegaBermudez, Johnson, \& Hsiao, 1991). We increased contact time so that our data could be more readily compared with other researchers' results.

At the start of testing, the number of trials in each session was 100. As testing continued, the number of trials was increased to 120 per session. All 26 letters of the alphabet were presented in random order with the constraint that the same letter could not be presented in two consecutive trials. No trial-by-trial feedback was provided. The decision not to use trial-by-trial feedback was in keeping with the procedure that Vega-Bermudez et al. (1991) used. These investigators did not use feedback, in part to examine asymmetries in letter identification performance. This issue is examined in the Discussion.

Training on the letter identification task was continued until a minimum of 2,600 trials had been collected from each subject. After this minimum was obtained, the subjects were tested again on the grating orientation task on the palm (Experiment 1). After the second set of grating orientation measurements was completed, 5 of the 6 subjects were tested again on letter identif ication, this time on the left palm. We were interested in whether there was transfer from the right (trained) palm to the left (untrained) palm. Subjects completed two sessions of letter identification on the left palm using the same procedure as had been used in testing the right palm.

\section{Results}

The performance on the letter identification task is shown in Figure 4 . The data are plotted by session. The change in duration from $250 \mathrm{msec}$ in earlier trials to $1,000 \mathrm{msec}$ in later trials is shown by the change in symbols in Figure 4. The change appears to have had little overall effect on performance. Subjects show steady improvement over sessions. Over the first eight sessions, the average improvement was $2.3 \%$ per session. This rate of improvement is less than the $4 \%$ per session improvement reported by Vega-Bermudez et al. (1991) using the fingerpad.
From these letter identification data, we wanted to get an estimate of performance that reflected spatial resolution. To get that estimate, we needed to consider the issue of the change in identification performance with practice. Our assumption was that the improvement in performance was due to perceptual learning, that subjects were becoming better able to focus on the critical features that differentiated the patterns, rather than changes in spatial resolution. For this reason we wanted to use asymptotic performance levels, instead of the average levels over the entire course of the experiment. For 3 of the 6 subjects, S2, S3, and S5, performance leveled off by the end of testing. For S1 and S4, performance may or may not be leveling off, and for S6, performance appears to be still improving. For Ss 1-5, performance over the last eight sessions was averaged, and these values are shown in Table 1. For S6, the last two sessions were averaged. It is possible that with continued testing subjects might improve and thus our estimate of letter recognition performance may be lower than these subjects might actually achieve. The estimate of asymptotic performance was $60 \%$ correct.

Five of the 6 subjects completed two blocks of trials using their left, untrained hand. Their performance averaged $46.9 \%$ correct as compared with their performance of $19.1 \%$ on the first two blocks of trials with their right hands. The difference in performance, $27.8 \%$, suggests that training at one location does lead to improved performance at other locations. The transfer is not perfect, however. The 5 subjects averaged $64.2 \%$ on their final two blocks of trials with their right hands.

\section{Discussion}

Are complex spatial patterns such as letters processed similarly by the fingerpad and palm? This question can be approached in several ways. First, one can ask whether spatial patterns that are easily identified when presented to the fingerpad are also easily identified when presented to the palm. To make this comparison we generated a confusion matrix for the letters presented to the palm, as shown in Figure 5. This confusion matrix could then be compared with results from the fingerpad. For the fingerpad, there were a number of matrices that might have been selected for purposes of comparison. A matrix from Loomis (1982) and a matrix from Vega-Bermudez et al. (1991) were selected to compare with the palm results. These were selected because they were based on a relatively large number of trials and because, in the latter case, the matrix had been more thoroughly analyzed than other matrices. To generate a confusion matrix on the palm with a level of performance similar to those achieved on the fingerpad (Loomis, 1982; Vega-Bermudez et al., 1991), the last 14 sessions for the palm data were used. The average performance on these sessions was $54.9 \%$ correct. The overall correlation between correct responses for the palm and fingerpad letters, the negative diagonal in the confusion matrices, was .85 with the Loomis data and .83 with the Vega-Bermudez et al. data. These correlations are similar-indeed somewhat higher-than the correlations 


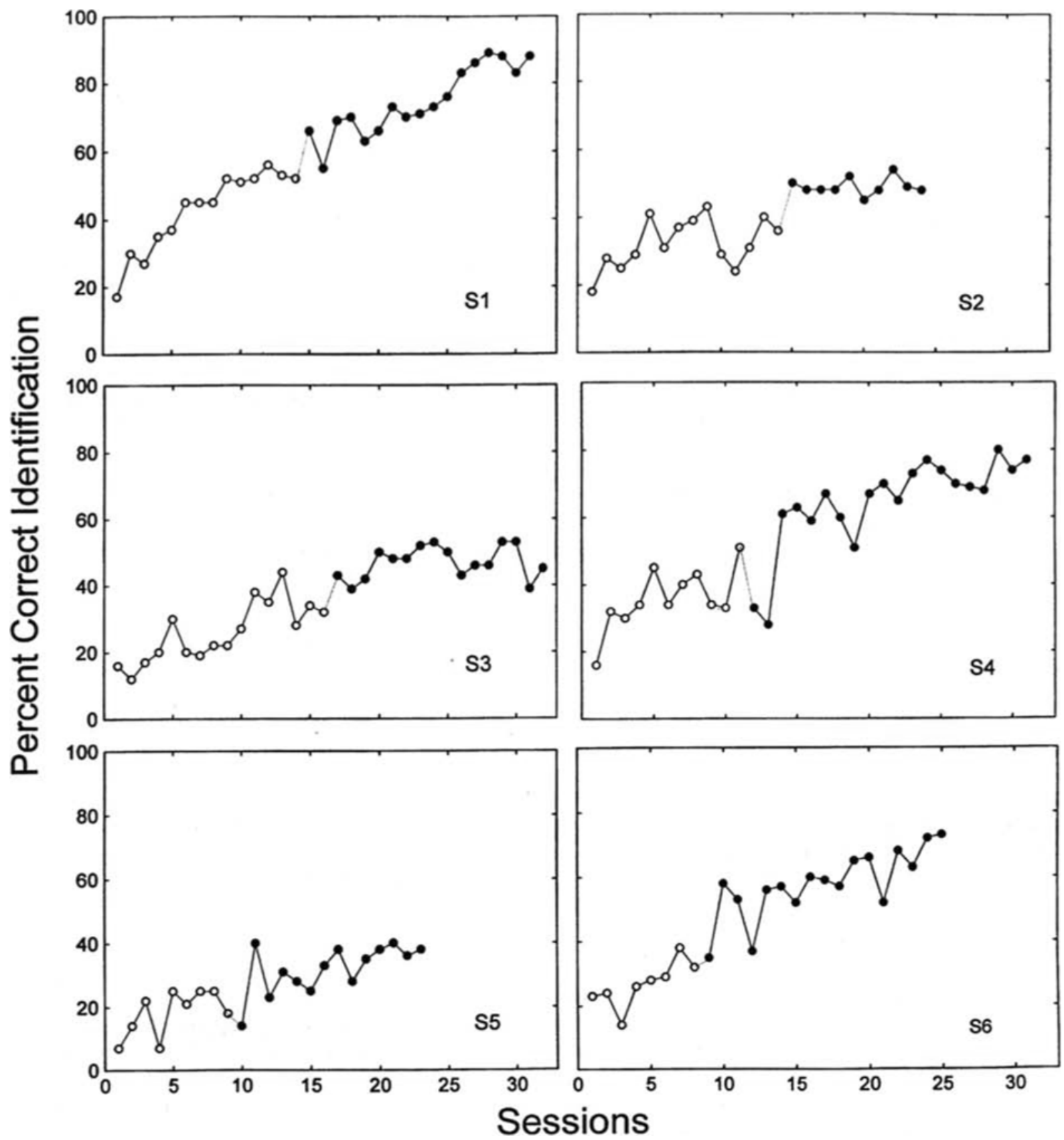

Figure 4. Percent correct letter identification as a function of training sessions. The results from each of 6 subjects, S1-S6, are shown. All subjects completed a minimum of 2,600 trials. The change in symbols from unfilled circles to filled circles indicates an increase in the duration of letter presentation from 250 to $1,000 \mathrm{msec}$.

obtained between several studies of letter identification on the fingerpad. The correlations ranged from .69 to .81 (Loomis, 1982). Thus, in terms of the relative difficulty in identifying the letters, there is nothing to suggest that spatial information is processed any differently on the palm than it is on the fingerpad.

Other comparisons are possible between the palm and fingerpad data. Vega-Bermudez et al. (1991) noted that the fingerpad confusion data were highly structured. Spe- cifically, a large number of the errors were concentrated on a small number of pairs of letters; half of all the errors were concentrated in 22 out of a possible 325 letter pairs, that is, $50 \%$ of the errors from $7 \%$ of the possible pairs. A similar result is seen with the palm data (Figure 5); $53 \%$ of the errors are concentrated in just $6 \%$ of the letter pairs. Vega-Bermudez et al. also observed that the confusions were highly asymmetric. For example, "C" was called "O" more than seven times more often than "O" 
Table 1

Asymptotic Letter Identification Performance

\begin{tabular}{cc}
\hline Subjects & Average Performance Final 8 Sessions \\
\hline S1 & 83 \\
S2 & 49 \\
S3 & 47 \\
S4 & 74 \\
S5 & 36 \\
S6 & $73^{*}$ \\
Overall average & 60 \\
\hline
\end{tabular}

*The percentage for S6 represents the subject's performance on the final two sessions.

was called "C." In the Vega-Bermudez et al. study, of the 22 pairs of letters that were most highly confused with one another (a mean confusion rate of $8 \%$ or greater), 15 of the 22 letters, $68 \%$, showed asymmetric confusions of 2.1:1 or more. For the palm data, there were 15 pairs of letters that averaged greater than $8 \%$ errors. The pairs are CG, CO, CQ, DO, GQ, GS, BR, GO, KX, MW, OQ, HN, VY, BE, and EZ. Of these 15, the first 11 listed showed an asymmetry of $2.1: 1$ or more, $73 \%$ of the 15 pairs. Also, 11 of these 15 letter pairs were among the 22 letter pairs from the fingerpad data.

Johnson and his co-workers have suggested that the performance on many spatial tasks is limited by the quality of the peripheral neural image. Vega-Bermudez et al. (1991) explained the nature of the letter confusions for the fingerpad data in terms of these neural images. The images are produced by recording activity from first-order afferents as raised letters are scanned across the fingerpad. Visual inspection of these images shows that a letter such as "C" evokes an image much like the letter "O" and, as noted, subjects often respond "O" when presented "C." Other asymmetries and confusions are explained in a similar fashion. A comparison of the 15 letter pairs with high rates of confusion (Figure 5) with the spatial event plots generated from the fingerpad (Vega-Bermudez et al., 1991, Figure 12) shows a number of pairs that might be explained by the nature of the peripheral image. Pairs such as $\mathrm{CO}, \mathrm{CQ}, \mathrm{GQ}, \mathrm{HN}, \mathrm{OQ}, \mathrm{VY}$, and BR produce similar neural images, and it is understandable why they would be confused with one another. On the other hand, the neural images for letter pairs such as FP and NR are similar to each other but do not result in high rates of confusion on the palm. The fact that the letters are scanned across the fingerpad to generate the neural images, whereas the letters presented to the palm were not scanned, likely accounts for some of the dissimilarities between the palm psychophysicaldata and the fingerpad neurophysiological results. The scanned images tend to produce strong neural responses to the leading edge of the letter but weaker responses to the trailing edge.

Vega-Bermudez et al. (1991) made an additional observation about the asymmetries between letter pairs that are confused with each other. They suggested that these confusions exist to a large extent because subjects did not receive trial-by-trial feedback. Given an unfamiliar task, identifying letters by means of touch, the subjects had to rely on some visual memory of the letters. Without feedback, subjects were unable to correct their misperceptions or to concentrate on those features of a letter that might distinguish it from a similar letter. VegaBermudez et al. predicted that if feedback were provided, the asymmetrical confusions would be greatly reduced. As part of another study in our laboratory, subjects received trial-by-trial feedback while attempting to identify letters presented to the palm. An analysis of the highly confused pairs of letters showed that, with feedback, the percentage of asymmetric pairs dropped to $50 \%$ as compared with $73 \%$ without feedback. Feedback reduced the number of asymmetrical pairs but did not eliminate them. We should also note that, although we have considered the asymmetries to be largely the result of perceptual similarities, there are other explanations for such asymmetries (Appelman \& Mayzner, 1982).

\section{GENERAL DISCUSSION}

How does letter identification performance on the palm, $60 \%$ correct, compare with letter identification performance on the fingerpad? Johnson and Phillips (1981) used letters ranging from 3 to $8 \mathrm{~mm}$ high. Their procedure was similar to ours in that (1) no lateral movement of the fingerpad was permitted, and (2) no trial-by-trial feedback was provided. Using their plot of probability correct as a function of letter height (Johnson \& Phillips, 1981, Figure 8), we determined that a letter $5.5 \mathrm{~mm}$ high would produce $60 \%$ correct letter identification on the fingerpad. A second study of letter recognition on the fingerpad featured a similar procedure (Phillips et al., 1983). From their data, we estimated that letters approximately $6.7 \mathrm{~mm}$ in height would yield $60 \%$ correct on the fingerpad. In other words, to achieve the same level of performance on the palm as the fingerpad required a pattern approximately 9.1 to 7.5 times larger $(50 \mathrm{~mm}$ to 5.5 or 50 to $6.7 \mathrm{~mm}$ ). Another study in which the conditions were fairly similar to those of the present study was that of Loomis (1981). Unlike in the present study, subjects were allowed to move their fingers in a slight circular pattern over the letter. With letters $7.2 \mathrm{~mm}$ high, subjects were correct on $56 \%$ of the trials. In this case the palm letters would be 6.9 times larger than the fingerpad. In a fourth study, in which the letters were scanned across the fingerpad, performance was $54.3 \%$ correct with 6-mm high letters (Vega-Bermudez et al., 1991). The ratio here would be $8.3: 1$ to produce a level of performance that is slightly below that of the present study.

The range of the ratios of letter sizes, 6.9 to 9.1 to 1 , corresponds to what one would expect on the basis of the density of innervation estimates for the SAI system, 8.8:1, and somewhat higher than that predicted by the RA system, 5.7:1. Recall that for the grating orientation task, the thresholds on the palm were 7.4 (pretesting) or 6.2 (posttesting) times the threshold on the fingerpad. These grating orientation results lead one to expect that the let- 


\section{Response}

\begin{tabular}{|c|c|c|c|c|c|c|c|c|c|c|c|c|c|c|c|c|c|c|c|c|c|c|c|c|c|c|c|}
\hline & A & B & C & D & $\mathrm{E}$ & $F$ & G & $\mathrm{H}$ & I & $\mathbf{J}$ & $\mathrm{K}$ & L & $\mathrm{M}$ & $\mathrm{N}$ & 0 & P & $\mathbf{Q}$ & $\mathrm{R}$ & $\mathrm{S}$ & $\mathrm{T}$ & U & V & W & $x$ & $Y$ & Z & $\mathrm{n}$ \\
\hline A & 71 & . & . & . & . & . & . & . & 6 & 8 & 1 & 5 & . & 1 & . & . & . & . & 1 & . & 1 & . & 1 & 1 & . & 1 & 422 \\
\hline B & 1 & 39 & 3 & 7 & 7 & 3 & 8 & 1 & . & 1 & 1 & 1 & 1 & 1 & 4 & 4 & 3 & 8 & 7 & . & . & . & 1 & . & . & 2 & 369 \\
\hline C & . & 2 & 32 & 5 & 2 & 1 & 21 & . & . & . & . & . & . & . & 17 & 2 & 12 & 1 & 2 & . & . & . & . & . & . & 1 & 408 \\
\hline D & 1 & 2 & 3 & 48 & . & . & 3 & 1 & . & . & . & 1 & 1 & . & 22 & 3 & 5 & 2 & 2 & . & 1 & . & . & 1 & . & 2 & 374 \\
\hline$E$ & . & 9 & 3 & 1 & 54 & 2 & 2 & 1 & 1 & 1 & 2 & . & . & 1 & . & 1 & . & 4 & 6 & . & . & . & 1 & 2 & 1 & 8 & 364 \\
\hline$F$ & 1 & 1 & . & . & 1 & 51 & . & . & . & 1 & 3 & . & & . & . & 1 & . & 3 & 3 & 5 & . & . & . & 2 & 5 & 4 & 377 \\
\hline G & 1 & 5 & 5 & 6 & 3 & 1 & 24 & 1 & . & . & . & . & . & . & 18 & . & 24 & 2 & 9 & . & . & . & . & 1 & . & 1 & 394 \\
\hline $\mathrm{H}$ & 1 & 3 & . & . & 1 & 1 & . & 44 & . & 1 & 3 & 1 & 6 & 19 & . & 1 & . & 1 & . & . & 8 & 4 & 3 & 1 & 1 & . & 385 \\
\hline I & . & . & . & . & . & . & . & . & 96 & . & . & 1 & . & . & . & . & . & . & . & . & 1 & . & . & . & 1 & . & 406 \\
\hline $\mathbf{J}$ & 4 & . & . & . & 1 & . & . & . & 1 & 81 & 1 & . & . & . & . & 1 & . & . & . & . & 1 & . & . & 1 & . & 1 & 367 \\
\hline $\mathrm{K}$ & . & 1 & 1 & . & 6 & 1 & . & 2 & . & . & 40 & . & 3 & 2 & . & . & . & 2 & . & . & 1 & 1 & 5 & 34 & 1 & 1 & 381 \\
\hline L & . & . & 3 & . & . & . & . & . & 2 & . & . & 91 & . & . & . & . & . & . & . & . & 2 & . & . & 1 & . & . & 395 \\
\hline$M$ & . & 1 & . & 1 & 1 & . & . & 8 & . & . & . & . & 56 & 6 & . & . & . & . & . & . & 5 & 1 & 21 & . & . & . & 377 \\
\hline $\mathrm{N}$ & 1 & 1 & . & 2 & 1 & . & . & 26 & 1 & 1 & 2 & . & 6 & 33 & . & . & . & . & . & . & 14 & 4 & 6 & 2 & . & . & 392 \\
\hline 0 & . & 2 & 4 & 5 & 1 & 1 & 8 & 1 & . & . & . & . & . & . & 68 & . & 9 & . & 1 & . & . & . & . & . & 1 & . & 379 \\
\hline$P$ & . & 3 &. & 9 & 1 & 3 & 1 & 1 & . & . & 1 & . & . & 1 & 3 & 66 & . & 2 & 2 & 3 & . & . & . & . & 2 & 1 & 376 \\
\hline $\mathrm{Q}$ & . & 2 & 5 & 7 & 1 & . & 8 & 2 & . & . & . & . & . & . & \begin{tabular}{|l|}
42 \\
\end{tabular} & 1 & 23 & 1 & 3 & . & . & . & . & . & . & 1 & 402 \\
\hline$R$ & 3 & 18 & 3 & 11 & 7 & 2 & 2 & 4 & . & 1 & 1 & . & 2 & 2 & 1 & 5 & 1 & 26 & 4 & 1 & 2 & 1 & . & 1 & 1 & 1 & 388 \\
\hline S & . & 8 & 6 & 4 & 6 & 1 & 23 & . & . & 1 & . & . & . & . & 7 & 5 & 7 & 2 & 25 & . & . & 1 & . & . & . & 2 & 381 \\
\hline $\mathrm{T}$ & . & . & . & . & . & 3 & . & 1 & . & . & 1 & . & 1 & 1 & . & 2 & . & 1 & . & 73 & . & 3 & . & 3 & 9 & 2 & 392 \\
\hline U & . & 1 & . & 5 & 1 & . & . & 4 & . & . & 1 & . & 3 & 2 & 2 & . & . & 1 & . & . & 68 & 1 & . & 1 & 1 & . & 386 \\
\hline V & . & 1 & . & . & . & 1 & . & 2 & . & . & 3 & . & 1 & 2 & . & . & . & . & . & 1 & 4 & 55 & 1 & 2 & \begin{tabular}{|l}
26 \\
\end{tabular} & . & 389 \\
\hline W & . & . & 1 & . & 1 & . & . & 4 & . & 1 & 2 & . & 6 & . & . & . & 1 & . & . & . & 2 & . & 82 & 1 & 1 & 1 & 398 \\
\hline$x$ & . & . & 1 & . & 3 & 1 & . & 2 & . & 1 & 10 & . & . & 2 & . & . & . & . & . & . & . & 4 & 1 & 61 & 4 & 8 & 419 \\
\hline$Y$ & 1 & . & 1 & . & . & 1 & . & 1 & . & 1 & 1 & . & . & 1 & . & 1 & . & . & . & 2 & 1 & \begin{tabular}{|l|}
27 \\
\end{tabular} & 1 & 3 & 60 & . & 369 \\
\hline z & 1 & 1 & 3 & 2 & 9 & 1 & . & . & . & 2 & 3 & 1 & . & . & . & . & . & 1 & 2 & 2 & . & . & . & 6 & 1 & 63 & 390 \\
\hline & 4 & 4 & 3 & 4 & 4 & 3 & 4 & 4 & 5 & 4 & 3 & 4 & 3 & 3 & 7 & 4 & 3 & 2 & 3 & 3 & 4 & 4 & 5 & 5 & 4 & 4 & 10080 \\
\hline & A & B & C & D & $E$ & $\mathrm{~F}$ & G & $\mathrm{H}$ & 1 & $\mathrm{~J}$ & $\mathrm{~K}$ & $\mathrm{~L}$ & $M$ & $\mathrm{~N}$ & 0 & $\mathrm{P}$ & $Q$ & $\mathrm{R}$ & $\mathrm{S}$ & $T$ & U & v & w & $x$ & $Y$ & z & \\
\hline
\end{tabular}

Figure 5. Pooled confusion matrix for 6 subjects. Data obtained using the right palm are presented. Each entry in the matrix, $p_{i j}$, represents the percentage of trials on which stimulus $S_{i}$ evoked the response $R_{j}$. The negative diagonal represents correct responses. Letter pairs with a mean confusion rate of $8 \%$ or greater are off-diagonal entries in boxes.

ters on the palm might have to be only 6.2 times as large as the letters on the fingerpad to achieve similar levels of performance. To put it another way, one might expect that the 50-mm letters would produce somewhat better performance than $60 \%$ correct. Given that continued training on the letter identification task might have led to improved performance, the grating orientation and letter identification results might be in closer agreement. Although it is not possible to predict precisely the grating orientation and letter identification results, the results are in the range of what is expected on the basis of the fingerpad psychophysical data and the neurophysiological results.

Both grating orientation and letter identification improved over time. The improvement in letter identification appears to be the result of practice with the letters. The average performance for the 6 subjects on the 1st day of testing was $16.2 \%$ correct. On the final day of testing, it was $61.5 \%$ correct. Is it possible that the changes in performance for both letter identification and grating orientation are the result of changes in underlying neural structures? Is the change in letter identification due to improved spatial resolution? Grating orientation thresh- olds dropped from 9.2 to $7.8 \mathrm{~mm}$ pre- to posttesting, an $18 \%$ improvement in sensitivity. Does an $18 \%$ improvement in spatial resolution correspond to a change in letter identification from $16 \%$ to $61 \%$ correct? On the basis of the Johnson and Phillips (1981) psychometric functions with letters presented to the fingerpad, it would require a fourfold increase in letter height to produce a $45 \%$ change in letter identification. If the change in performance were the result of a change in spatial resolution, the change in resolution should be on the order of a fourfold change rather than the $18 \%$ that was observed. In short, the improvement in letter identification with experience appears not to be the result of a change in spatial resolution.

Improvement in letter identification has also been observed with patterns presented to the fingerpad, at least in some studies. As noted, Vega-Bermudez et al. (1991) reported an improvement of about 4\% per session (104 trials per session) for eight sessions. After eight sessions, performance leveled off. In the present study, performance improved at a rate of $2.3 \%$ per session for the first eight sessions. The overall improvement was $45.3 \%$ over an average of 27.7 sessions, a $1.6 \%$ increase per session. 
Vega-Bermudez et al. attributed the improvement in performance to perceptual learning, a view shared by other investigators (e.g., Sathian, 1998). The fact that the changes occur over a relatively long training period is consistent with perceptual learning, although it should be noted that long-lasting changes are also consistent with high-level cognitive learning (Goldstone, 1998). No trial-by-trial feedback was used, so the process of improvement presumably relied on the differentiation of the structure inherent in the letters.

One of the questions that arises in studies that have shown changes that might be attributed to perceptual learning is, how specific is the learning? Is it specific to a particular site? For tactile stimuli, the answer has been mixed, with some tasks showing a high degree of specificity and others less so (Sathian, 1998). In the present study, the fact that performance on the left, untrained hand was substantially better than that seen in the first sessions on the right hand suggests that the learning is not site specific. The transfer from the right to the left hand was not complete, however. These findings are similar to the results from an earlier study in the laboratory, in which subjects were trained to identify letters presented to the left palm and then tested on the right palm. Whether one chooses to call the improvements in performance with experience a form of perceptual learning or cognitive learning, there appears to be substantial transfer of this task between sites.

Also relevant to the issue of perceptual learning is the change in sensitivity on the grating orientation task. The improvement from pre- to posttesting is unlikely to be the result of experience with the grating orientation task. The pretesting threshold was determined over four sessions. There was no change in performance from the first to the fourth session. The next testing session was at the end of the letter identification sessions, and on this session subjects showed a substantial improvement in sensitivity. It appears that the experience with letter identification resulted in improvement in grating orientation sensitivity. Previous measurements on the fingerpad have shown no improvement with experience (Craig \& Kisner, 1998; Van Boven \& Johnson, 1994a). It is unlikely that training with letters improves grating orientation sensitivity more than training with grating orientation. It is more likely that the palm is different from the fingerpad with regard to experience and grating orientation sensitivity. Grant, Thiagarajah, and Sathian (2000) have discussed the possibility that visual imagery aids the grating orientation task. The subject's sense of a distal-proximal axis on the fingerpad may be more strongly developed than it is on the palm. Repeated experience with letters that have strong vertical axes such as I, T, L, and J, and so forth, may help subjects develop a clearer sense of the proximaldistal and lateral-medial axes on the palm. Consistent with this view is the finding that repeated testing of grating orientation on the lip, an area that might also be expected to lack highly developed spatial axes, also produced a significant improvement in performance (Van Boven \& Johnson, 1994a).

\section{REFERENCES}

Appelman, I. B., \& Mayzner, M. S. (1982). Application of geometric models to letter recognition: Distance and density. Journal of Experimental Psychology: General, 111, 60-100.

Bolanowski, S. J., Gescheider, G. A., Verrillo, R. T., \& CheckOSKY, C. M. (1988). Four channels mediate the mechanical aspects of touch. Journal of the Acoustical Society of America, 84, 1680-1694.

Craig, J. C. (1999). Grating orientation as a measure of tactile spatial acuity. Somatosensensory \& Motor Research, 16, 197-206.

Craig, J. C., \& Johnson, K. O. (2000). The two-point threshold: Not a measure of tactile spatial resolution. Current Directions in Psychological Science, 9, 29-32.

Craig, J. C., \& Kisner, J. M. (1998). Factors affecting tactile spatial acuity. Somatosensensory \& Motor Research, 15, 29-45.

Darian-Smith, J., \& Kenins, P. (1980). Innervation density of mechanoreceptive fibres supplying the glabrous skin of the monkey's index finger. Journal of Physiology, 309, 147-155.

Geldard, F. A. (1960). Some neglected possibilities of communication. Science, 131, 1583-1588.

Goldstone, R. L. (1998). Perceptual learning. Annual Review of Psychology, 49, 585-612.

Grant, A. C., Thiagarajah, M. C., \& Sathian, K. (2000). Tactile perception in blind Braille readers: A psychophysical study of acuity and hyperacuity using gratings and dot patterns. Perception \& Psychophysics, 62, 301-312.

Greenspan, J. D., \& Bolanowski, S. J. (1996). The psychophysics of tactile perception and its peripheral physiological basis. In L. Kruger (Ed.), Pain and touch (2nd ed., pp. 25-103). San Diego: Academic Press.

Johansson, R. S., \& Vallbo, A. B. (1979). Tactile sensibility in the human hand: Relative and absolute densities of four types of mechanoreceptive units in glabrous skin. Journal of Physiology, 286, 283-300.

Johnson, K. O., \& HsiaO, S. S. (1992). Tactual form and texture perception. Annual Review of Neuroscience, 15, 227-250.

Johnson, K. O., Hsiao, S. S., \& Twombly, I. A. (1995). Neural mechanisms of tactile form recognition. In M. Gazzaniga (Ed.), The cognitive neurosciences (pp. 253-267). Cambridge, MA: MIT Press.

Johnson, K. O., \& Phillips, J. R. (1981). Tactile spatial resolution: I. Two-point discrimination, gap detection, grating resolution, and letter recognition. Journal of Neurophysiology, 46, 1177-1191.

Johnson, K. O., Van Boven, R. W., \& Hsiao, S. S. (1994). The perception of two points is not the spatial resolution threshold. In J. Boivie, P. Hansson, \& U. Lindblom (Eds.), Touch, temperature, and pain in health and disease: Mechanisms and assessments, progress in pain research and management (Vol. 3, pp. 389-404). Seattle, WA: IASP Press.

Loomis, J. M. (1981). Tactile pattern perception. Perception, 10, 5-27. Loomis, J. M. (1982). Analysis of tactile and visual confusion matrices. Perception \& Psychophysics, 31, 41-52.

Loomis, J. M. (1990). A model of character recognition and legibility. Journal of Experimental Psychology: Human Perception \& Performance, 16, 106-120.

Loomis, J. M., \& Lederman, S. J. (1986). Tactual perception. In K. R. Boff, L. Kaufman, \& J. P. Thomas (Eds.), Handbook of perception and human performance (pp. 1-41). New York: Wiley.

Patel, J., Essick, G. K., \& Kelly, D. G. (1997). Utility of square-wave gratings to assess perioral spatial acuity. Journal of Oral Maxillofacial Surgery, 55, 593-601.

Phillips, J. R, \& Johnson, K. O. (1981). Tactile spatial resolution: II. Neural representation of bars, edges, and gratings in monkey primary afferents. Journal of Neurophysiology, 46, 1192-1203.

Phillips, J. R., Johnson, K. O., \& Browne, H. M. (1983). A comparison of visual and two modes of tactual letter resolution. Perception \& Psychophysics, 34, 243-249.

Sathian, K. (1998). Perceptual learning. Current Science, 75, 451-457. 
Sathian, K., \& Zangaladze, A. (1996). Tactile spatial acuity at the human fingertip and lip: Bilateral symmetry and interdigit variability. Neurology, 46, 1464-1466.

Sathian, K., Zangaladze, A., Green, J., Vitek, J. L., \& DeLong, M. R. (1997). Tactile spatial acuity and roughness discrimination: Impairments due to aging and Parkinson's disease. Neurology, 49, 168-177.

VAn Boven, R. W., \& Johnson, K. O. (1994a). The limit of tactile spatial resolution in humans: Grating orientation discrimination at the lip, tongue, and finger. Neurology, 44, 2361-2366.

VAn Boven, R. W., \& Johnson, K. O. (1994b). A psychophysical study of the mechanisms of sensory recovery following nerve injury in humans. Brain, 117, 149-167.
Vega-Bermudez, F., Johnson, K. O., \& Hsiao, S. S. (1991). Human tactile pattern recognition: Active versus passive touch, velocity effects, and patterns of confusion. Journal of Neurophysiology, $\mathbf{6 5}$, 531-546.

Weber, E. H. (1996). De tactu. In H. E. Ross \& D. J. Murray (Eds. and Trans.), E. H. Weber on the tactile senses (2nd ed., p. 49). Hove, U.K.: Erlbaum. (Original work published 1834)

(Manuscript received September 8, 1999; revision accepted for publication April 18, 2000.) 